\title{
Frostbite-like Skin Lesion as an Autonomic Symptom of Isaacs' Syndrome
}

\author{
Hikaru Doi ${ }^{1}$, Kimiyoshi Arimura ${ }^{2}$, Yasumasa Ohyagi ${ }^{1}$ and Jun-ichi Kira ${ }^{1}$
}

\begin{abstract}
A 25-year-old woman complained of numbness of the extremities, following muscle rigidity and tenderness. The presence of anti-voltage-gated potassium channel antibody led to the diagnosis of Isaacs' syndrome. Twenty-seven months after the first symptom, she developed a pricking pain sensation in the lateral left foot, and then gradually developed a purple skin lesion resembling frostbite. The lesion completely disappeared 2 days later. An incidental episode occurred at the same site 8 months later. Frostbite-like skin lesions may be a rare autonomic manifestation in Isaacs' syndrome.
\end{abstract}

Key words: Isaacs' syndrome, frostbite, skin lesion, anti-voltage-gated potassium channel antibody

(Intern Med 50: 1113-1115, 2011)

(DOI: 10.2169/internalmedicine.50.4998)

\section{Introduction}

Isaacs' syndrome is a disease characterized by muscle rigidity and painful muscle contractions in the presence of anti-voltage-gated potassium channel (VGKC) antibody produced in an auto-immune or paraneoplastic condition $(1,2)$. Among autonomic symptoms, hyperhidrosis is occasionally seen but other autonomic symptoms are quite rare (3). Here, we report a rare case presenting focal skin lesions probably due to peripheral autonomic dysfunction.

\section{Case Report}

A 25-year-old woman first developed fatigue of the lower extremities with numbness and muscle twitching in both thighs, especially after exercise and alcohol drinking. She also had nocturnal sweating, even in the winter season. Neurological examination showed an increased deep tendon reflex in both lower extremities. Fasciculation and myokymia of both thighs were observed after she took a bath. Laboratory data showed positive anti-nuclear antibody (titer of 1: 160), low CH50 (18 U/mL), and decreased white blood cells $(3,200 / \mu \mathrm{L})$, suggesting an autoimmune condition. Total cell counts $(0$ cells $/ \mu \mathrm{L})$, total protein amounts $(12 \mathrm{mg} / \mathrm{dL})$, and glucose levels $(58 \mathrm{mg} / \mathrm{dL})$ in the cerebrospinal fluid were within normal limits. Brain and spinal magnetic resonance imaging disclosed no abnormalities. Nerve conduction study revealed prolonged duration of $\mathrm{F}$ waves in both tibial and peroneal nerves, together with stimulus-induced repetitive discharge (SIRD). Needle electromyography revealed spontaneous motor unit potential discharges and short interval repetitive discharges in the vastus medialis muscle at rest, compatible with fasciculation and myokymia, respectively. Computed tomography and ultrasonography in neck, thorax, abdomen, and pelvis showed no abnormalities. Following detection of $229 \mathrm{pM}$ anti-VGKC antibody in serum (normal control $<100 \mathrm{pM}$ ), we diagnosed her as having Isaacs' syndrome. Administration of phenytoin (200 mg/day) and gabapentin $(1,800 \mathrm{mg} /$ day $)$ and plasma absorption therapy ameliorated her symptoms and duration of SIRD in both tibial and peroneal nerves was decreased.

Twenty-seven months after the initial symptom, she suddenly noticed a strong pain in the outer aspect of her left foot after taking a bath. The skin color gradually turned purple and the lesion resembled frostbite (Fig. 1). The tenderness continued all night and the following day, and she could not wear shoes because of the tenderness. The skin lesion completely disappeared 2 days after onset. Eight months after the first episode, she noticed discomfort of the

${ }^{1}$ Department of Neurology, Neurological Institute, Graduate School of Medical Sciences, Kyushu University, Japan and ${ }^{2}$ Department of Neurology, Okatsu Hospital, Japan

Received for publication December 14, 2010; Accepted for publication January 24, 2011

Correspondence to Dr. Jun-ichi Kira, kira@neuro.med.kyushu-u.ac.jp 


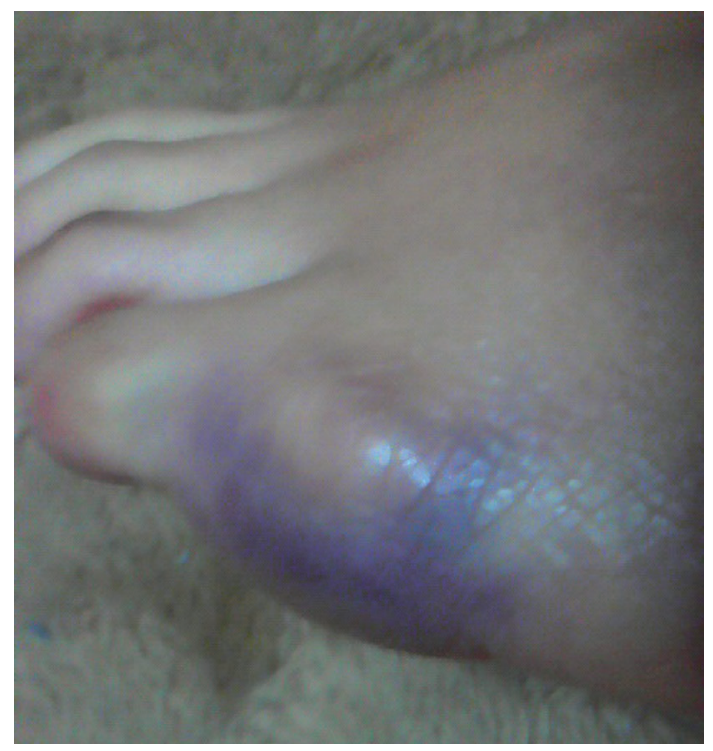

Figure 1. A focal skin lesion with pain on the outer side of the left foot. Note the purple discoloration of the skin lesion.

left foot while walking. She gradually noticed pain of the left foot and a skin color change just like the first episode. The skin lesion again disappeared after 2 days. When the skin lesion appeared, her neuromyotonia symptoms were not exacerbated. Neurological examination after the appearance of skin lesions indicated no exacerbation of neurological symptoms. The positive anti-nuclear antibody, low CH50, and decreased white blood cell count detected at the first examination were not observed during the 2-year observation period, and no other auto-antibodies were detected during the entire course of the disease.

\section{Discussion}

Isaacs' syndrome is an antibody-mediated potassium channel disorder characterized by muscle cramp and slow relaxation following muscle contraction and autoantibodies against VGKC, a dendrotoxin-sensitive fast potassium channel. Autoantibody-mediated VGKC dysfunction leads to hyperexcitability of the peripheral nerves $(1,2)$. With regard to central nervous system (CNS) involvement, anti-VGKC antibody is detected in Morvan syndrome, limbic encephalitis, and temporal lobe epilepsy, which manifest neurologic, psychiatric, and autonomic impairments such as REM sleep behavior disorder, hallucination, epilepsy, and tachycardia (3). Furthermore, contactin-associated protein-2 has been identified as one of the major targets of potassium channel antibodies in acquired neuromyotonia and Morvan syndrome (4). Regarding peripheral nerve involvement, muscle cramp, twitching, and stiffness are the main manifestations. Hyperhidrosis is the most common autonomic symptom. Coexistence of central and peripheral nervous system symptoms in the same patient is quite rare except for Morvan syndrome (5).

The autonomic symptoms in Morvan syndrome are thought to be of CNS origin, and those originating from peripheral autonomic nerves are rare except for hyperhidrosis (6). A rare case of Isaacs' syndrome with urinary incontinence has been reported (7). Concerning skin lesions in Isaacs' syndrome, there are only two reported cases with complex regional pain syndrome-like symptoms as the first manifestation of Isaacs' syndrome $(8,9)$. In these cases, numbness and pain in the extremities, edema of the distal portions of the extremities, muscular atrophy, and reduced skin temperature were found.

In the present case, the focal skin lesion with pain resembled frostbite and completely disappeared within 2 days. Although other autoimmune diseases, such as systemic lupus erythematosus and dermatomyositis, sometimes exist in Isaacs' syndrome, the present case had no clinically overt autoimmune diseases. Therefore, although we could not completely rule out Raynaud's phenomenon associated with connective tissue diseases, we assume that the skin lesion in the present case was related to Isaacs' syndrome and that blood congestion in the capillaries was the main cause of the skin lesion. The anti-VGKC antibody may have caused dysfunction of the vasoactive peripheral autonomic nerves. The fact that the skin lesions and pain were not accompanied by exacerbation of neuromyotonia symptoms implies that the anti-VGKC antibody affects peripheral autonomic nerves independent from peripheral motor nerves, suggesting the presence of small fiber-dominant manifestations in the present case. We therefore propose that the frostbite-like skin lesion is a rare peripheral autonomic manifestation in Isaacs' syndrome.

The authors state that they have no Conflict of Interest (COI).

\section{References}

1. Isaacs H. Progress in the diagnosis of painful limb syndromes. S Afr Med J 39: 618-621, 1965.

2. Sinha S, Newsom-Davis J, Mills K, Byrne N, Lang B, Vincent A. Autoimmune aetiology for acquired neuromyotonia (Isaacs' syndrome). Lancet 338: 75-77, 1991.

3. Tan KM, Lennon VA, Klein CJ, Boeve BF, Pittock SJ. Clinical spectrum of voltage-gated potassium channel autoimmunity. Neurology 70: 1883-1890, 2008.

4. Irani SR, Alexander S, Waters $\mathrm{P}$, et al. Antibodies to Kv1 potassium channel-complex proteins leucine-rich, glioma inactivated 1 protein and contactin-associated protein-2 in limbic encephalitis, Morvan's syndrome and acquired neuromyotonia. Brain 133: 2734-2748, 2010.

5. Hart IK, Maddison P, Newsom-Davis J, Vincent A, Mills KR. Phenotypic variants of autoimmune peripheral nerve hyperexcitability. Brain 125: 1887-1895, 2002.

6. Liguori R, Vincent A, Clover L, et al. Morvan's syndrome: peripheral and central nervous system and cardiac involvement with antibodies to voltage-gated potassium channels. Brain 124: 24172426, 2001.

7. Halbach M, Hömberg V, Freund HJ. Neuromuscular, autonomic and central cholinergic hyperactivity associated with thymoma and acetylcholine receptor-binding antibody. J Neurol 234: 433-436, 1987. 
Intern Med 50: 1113-1115, 2011 DOI: 10.2169/internalmedicine.50.4998

8. Watanabe O, Arimura K, Takada R, Michizono K, Takashima H. Study of numbness and pain in immune-mediated $\mathrm{K}^{+}$channel disease. In: 22nd Congress of the Japanese Society for Neuroimmunology. Tokyo, Japan, 2010: 17-19.
9. Yoshioka H, Onodera H, Saito T, et al. Autonomic dysfunction in Isaacs syndrome: A case with asymmetrical sympathetic stimulation. Eur J Neurol 14: S227-S228, 2007.

(C) 2011 The Japanese Society of Internal Medicine http://www.naika.or.jp/imindex.html 